\section{Magnetic Field-Responsive Pulsatile Drug Release Using A Magnetic Fluid}

\author{
Chihiro Takei, ${ }^{*}, a$ Kenji Mori, ${ }^{a}$ Takeshi Oshizaka, ${ }^{a}$ and \\ Kenji Sugibayashi*,a,b
}

${ }^{a}$ Faculty of Pharmaceutical Sciences, Josai International University; 1 Gumyo, Togane, Chiba 283-8555, Japan: and ${ }^{b}$ Faculty of Pharmacy and Pharmaceutical Sciences, Josai University; 1-1 Keyakidai, Sakado, Saitama 350-0295, Japan.

Received August 19, 2021; accepted October 31, 2021

Ferrofluids are colloidal liquids with fine magnetic particles. They change shape and fluidity depending on the magnitude and direction of the external magnetic field. The magnetic field-responsive pulsatile release of a model drug, lidocaine hydrochloride (LID·HCI), was determined using a depot-type injection containing white petrolatum and/or hydrophilic cream with a magnetic fluid in various proportions. Drug release was confirmed using a self-made diffusion cell and the application of a moving magnet at the bottom of the preparation. Magnetic field-responsive LID release was observed only when using the white petrolatum preparation and depended on the concentration of the magnetic fluid. Magnetic field responsiveness was not observed in the preparation with only the hydrophilic cream. A greater magnetic field-responsive release was observed with a combination of white petrolatum and hydrophilic cream than with white petrolatum alone. These results may lead to the development of an injectable formulation that enables pulsatile administration of macromolecular drugs.

Key words magnetic fluid; pulsatile release; magnetic fieldresponsive release; depot formulation; hydrophilic drug

\section{Introduction}

Recently, the focus of drug development has shifted from small molecular drugs to macromolecular biopharmaceuticals. This is because small molecular drug seeds are being depleted and the targets of drug development have changed from lifestyle-related diseases to cancers, autoimmune diseases, and infectious diseases. However, it can be difficult to prepare oral dosages of macromolecular drugs because of their malabsorption through the gastrointestinal tract. Therefore, they must be injected. This places a heavy burden on the patients. Interferon and teriparatide acetate injections require weekly outpatient administration; insulin and somatropin self-injections require new needles for each injection; administration of these drugs can be especially difficult for children and older patients. Moreover, vaccinated individuals sometimes require booster shots to achieve sufficient immunity. These examples demonstrate the need for a simple and easy injection preparation for pulsatile drug release.

Here, we utilized a ferrofluid and an injection preparation containing a magnetic fluid. This functions as a pulsatile release preparation for the delivery of therapeutic agents in a single injection. Ferrofluids are colloidal liquids in which fine magnetic particles such as magnetite $\left(\mathrm{Fe}_{3} \mathrm{O}_{4}\right)$ are coated with a surfactant and dispersed throughout the medium. They are al- ready being used in the medical field for drug carrying, imaging, and treating hyperthermia. ${ }^{1-3)}$ We focused on the fact that the drug on the formulation surface is only released when a water-soluble drug powder with a variety of molecular weights is encapsulated in a hydrocarbon-based vehicle such as white petrolatum. We hypothesized that with the addition of a magnetic fluid the release of a therapeutic drug from a depot-type injection containing white petrolatum and/or hydrophilic cream may be controlled by an external magnet.

In this study, various materials were combined to prepare test depot preparations, and the in vitro release properties of a model hydrophilic compound, such as lidocaine hydrochloride $(\mathrm{LID} \cdot \mathrm{HCl})$ were tested using a self-made diffusion cell.

\section{Experimental}

Materials and Model Depot Preparation Ferrofluid DS-60 was purchased from Sigma High Chemical Co., Ltd. (Chigasaki, Kanagawa, Japan). JP grade white petrolatum and hydrophilic cream were used as injection vehicles. LID $\cdot \mathrm{HCl}$ was purchased from FUJIFILM Wako Pure Chemical Corporation (Osaka, Japan). Other chemicals were commercially available, special grade products.

We prepared four types of depot preparations (Rp. 1-4). The $\mathrm{LID} \cdot \mathrm{HCl}$ concentration was set to $1 \%$ for the white petrolatum (Rp. 1, 2), hydrophilic cream (Rp. 3), and a 1:1 mixture of both (Rp. 4). The magnetic fluid concentration was set to $30 \%$ for Rp. $2-4$ and $15 \%$ for Rp. 1 .

In Vitro Drug Release Test The release properties of LID $\cdot \mathrm{HCl}$ from the pharmaceutical product were evaluated using a self-made acrylic diffusion cell (Fig. 1). The diffusion cell was composed of a lower columnar part A (diameter $46 \mathrm{~mm}$, depth $1 \mathrm{~mm}$ ) for setting the formulation and an upper double columnar part B with a receiver solution on the inside and water on the outside for heat retention. Part A was filled with the test product, covered with a dialysis membrane (MWCO: 3500), and fixed together with part B. P dissolution test 1 st solution $\left(37^{\circ} \mathrm{C}, \mathrm{pH} 1.2,100 \mathrm{~mL}\right)$ was placed inside part $\mathrm{B}$, and water $\left(37^{\circ} \mathrm{C}\right)$ was placed in the outer column. The

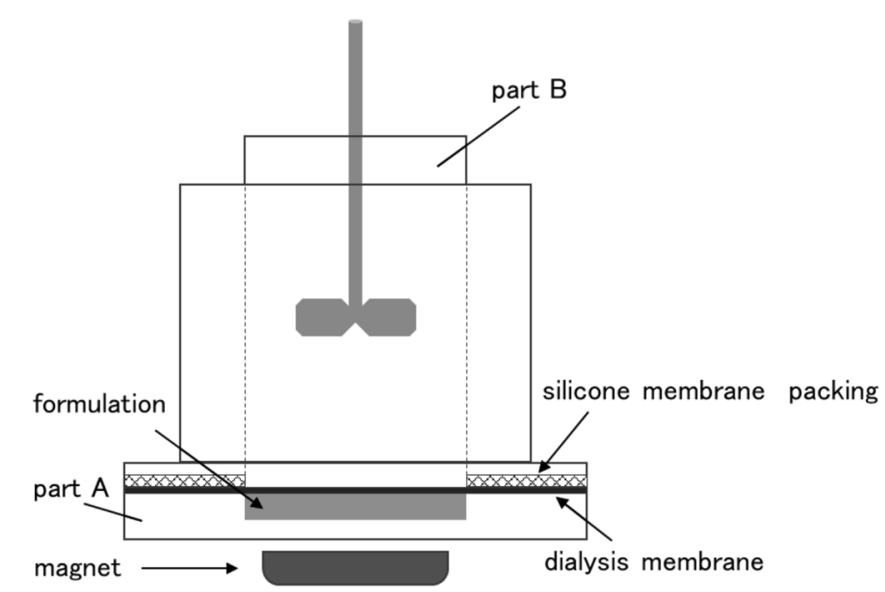

Fig. 1. Overview of Self-made Diffusion Cell

Dialysis membrane was used to determine the release of LID $\cdot \mathrm{HCl}$ from the formulation. Silicone membrane packing with a hollowed circular center (diameter of hollowed circle: $46 \mathrm{~mm}$ ) was used to ensure tight adhesion between parts A and B. 
(a)

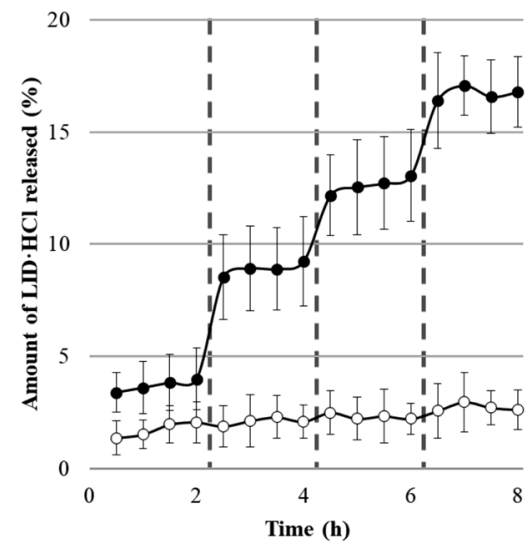

(b)

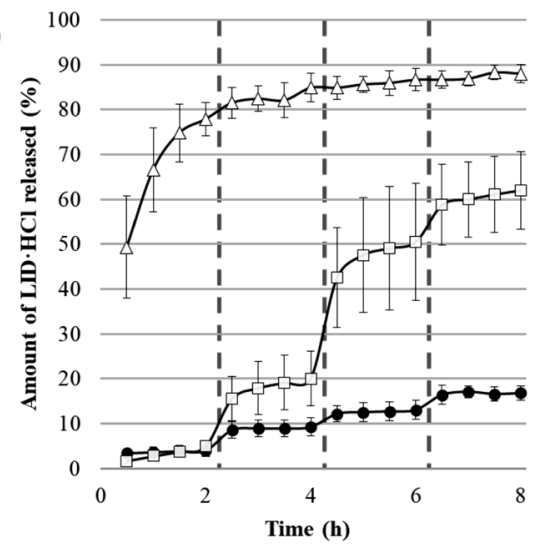

Fig. 2. Cumulative Amount of LID $\cdot H C l$ Released from Different Vehicles

a) Effect of magnetic fluid concentration on the release of LID $\cdot \mathrm{HCl}$ from white petrolatum. Rp. 1 (magnetic fluid concentration, $15 \%$, $\bigcirc$ ) and Rp. 2 (magnetic fluid concentration, $30 \%$, ). b) Effect of the formulation vehicle on the release of LID $\cdot \mathrm{HCl}$ at a magnetic fluid concentration of $30 \%$. Rp. 2 (white petrolatum, ), Rp. 3 (hydrophilic cream, $\triangle$ ), and Rp. 4 (1:1 mixture of white petrolatum and hydrophilic cream, $\square)$. Data are expressed as the means \pm standard deviation (S.D.) of 3-6 experiments.

receiver solution was stirred with a propeller $(100 \mathrm{rpm})$, and the water was warmed in a constant-temperature bath and circulated using an electric pump. The receiver solution was sampled over time, and the magnet (magnetic flux density: $0.37 \mathrm{~T}$ ) was periodically moved to the bottom of the diffusion cell for $1 \mathrm{~min}$. The $\mathrm{LID} \cdot \mathrm{HCl}$ in the receiver solution was quantified using HPLC.

\section{Results and Discussion}

Figure 2 shows the time course of LID $\cdot \mathrm{HCl}$ release from the depot formulations and the effect of the magnetic fluid on it. No change was observed in the amount of $\mathrm{LID} \cdot \mathrm{HCl}$ released for $\mathrm{Rp}$. 1 (white petrolatum alone, magnetic fluid $=15 \%$ ), even when a magnetic field was applied, with less than $5 \%$ of the applied amount released over $8 \mathrm{~h}$ (Fig. 2a, $\bigcirc)$. In contrast, pulsatile release of $\mathrm{LID} \cdot \mathrm{HCl}$ was observed in Rp. 2 immediately after application of the magnetic field (white petrolatum alone, magnetic fluid $=30 \%$ ), and the response to the magnetic field increased depending on the concentration of the magnetic fluid (Figs. 2a and b, O). The increase in the release of $\mathrm{LID} \cdot \mathrm{HCl}$ was caused by the movement of the magnetic fluid in the formulation in response to the magnetic field. The $\mathrm{LID} \cdot \mathrm{HCl}$ powder moved from inside the preparation to the surface. Figure $2 b$ shows the effect of different vehicles on $\mathrm{LID} \cdot \mathrm{HCl}$ release. In Rp. 3 (hydrophilic cream alone), approximately $50 \%$ of $\mathrm{LID} \cdot \mathrm{HCl}$ was released immediately after beginning the release test, followed by a slow and gradual release without responding to the magnetic field (Fig. 2b, $\triangle$ ). For Rp. 4 (1:1 mixture of white petrolatum and hydrophilic cream), the $\mathrm{LID} \cdot \mathrm{HCl}$ release rate immediately after beginning the test was similar to Rp. 2. However, pulsatile release was observed when a magnetic field was applied (Fig. 2b, $\square$ ), and the amount of $\mathrm{LID} \cdot \mathrm{HCl}$ released was much higher than that of Rp. 2.

These differences in release characteristics maybe due to the polarities of the formulation vehicles. Hydrophilic $\mathrm{LID} \cdot \mathrm{HCl}$ is insoluble in white petrolatum (white petrolatum is insoluble in water); therefore, the receiver solution was not able to enter the formulation. As a result, only the $\mathrm{LID} \cdot \mathrm{HCl}$ powder in contact with the receiver solution was dissolved and released when the magnetic preparation was magnetically stirred.
In contrast, $\mathrm{LID} \cdot \mathrm{HCl}$ partially dissolved in the hydrophilic cream (hydrophilic cream can dissolve in water), the receiver solution could enter the depot formulation. As a result, no effect was observed with the application of a magnetic field. The LID $\cdot \mathrm{HCl}$ moved through the formulation, resulting in a constant release immediately after adding the receiver solution. Moreover, when the white petrolatum-hydrophilic cream mixture was used, the hydrophilic cream containing $\mathrm{LID} \cdot \mathrm{HCl}$ was probably dispersed throughout the white petrolatum. The application of a magnetic field brought the hydrophilic cream in contact with the receiver solution to release $\mathrm{LID} \cdot \mathrm{HCl}$.

The obtained results suggest that the entrapment of magnetic fluid in white petrolatum or a mixture of white petrolatum and hydrophilic cream enables the magnetically responsive pulsatile release of a water-soluble drug like LID $\cdot \mathrm{HCl}$. Although only in vitro data are shown in this study, we have already obtained preliminary in vivo data on the magnetically responsive release of drugs from subcutaneously implanted preparations in rats. We also found that the drug preparation containing magnetic fluid did not move from the injected subcutaneous tissue in rats, suggesting that it may be easy to remove the formulation from the body when the drug is no longer released. Moreover, this type of magnetic field-responsive pulsatile drug release system may also be applicable to medium- and high-molecular-weight biopharmaceuticals and antigens. Thus, the present concept may be a viable means of pulsatile drug release from a single injection.

Conflict of Interest The authors declare no conflict of interest.

\section{References}

1) Alexiou C., Jurgons R., Schmid R., Hilpert A., Bergemann C., Parak F., Iro H., J. Magn. Magn. Mater., 293, 389-393 (2005).

2) Jordan A., Scholz R., Wust P., Fähling H., Krause J., Wlodarczyk W., Sander B., Vogl Th., Felix R., Int. J. Hyperthermia, 13, 587-605 (1997).

3) Park J. H., Cho H. J., Yoon H. Y., Yoon I. S., Ko S. H., Shim J. S., Cho J. H., Park J. H., Kim K., Kwon I. C., Kim D. D., J. Control. Release, 174, 98-108 (2014). 